\title{
Design and characterization of a quantum heat pump in a driven quantum gas
}

\author{
Arko Roy ${ }^{1,2, *}$ and André Eckardt ${ }^{1,3, \dagger}$ \\ ${ }^{1}$ Max-Planck-Institut für Physik komplexer Systeme, Nöthnitzer Straße 38, 01187 Dresden, Germany \\ ${ }^{2}$ INO-CNR BEC Center and Dipartimento di Fisica, Università di Trento, 38123 Trento, Italy \\ ${ }^{3}$ Technische Universität Berlin, Institut für Theoretische Physik, Hardenbergstraße 36, 10623 Berlin, Germany
}

(Received 16 December 2019; accepted 16 March 2020; published 9 April 2020)

\begin{abstract}
We propose the implementation of a quantum heat pump with ultracold atoms. It is based on two periodically driven coherently coupled quantum dots using ultracold atoms. Each dot possesses two relevant quantum states and is coupled to a fermionic reservoir. The working principle is based on energy-selective driving-induced resonant tunneling processes, where a particle that tunnels from one dot to the other either absorbs or emits the energy quantum $\hbar \omega$ associated with the driving frequency, depending on its energy. We characterize the device using Floquet theory and compare simple analytical estimates to numerical simulations based on the Floquet-Born-Markov formalism. In particular, we show that driving-induced heating is directly linked to the micromotion of the Floquet states of the system.
\end{abstract}

DOI: 10.1103/PhysRevE.101.042109

\section{INTRODUCTION}

The miniaturization of heat engines and pumps to systems consisting of a few relevant quantum states only [1,2] and their description in terms of quantum thermodynamics [3-6] constitutes a fascinating and active field of research. In this context the implementation and investigation of such devices with ultracold neutral atoms in tailored light-shift potentials defines a promising direction of research. Especially the recently developed quantum-gas microscopes, where digital mirror devices are employed for microstructuring almost arbitrary potential landscapes with high resolution both in space and time [7-12], provide an interesting platform for this goal. One advantage of atomic quantum gases in optical potentials is that they provide extremely clean conditions for studying the fundamental properties of quantum engines and pumps, since they do not suffer from dissipation induced by the coupling to phonons or due to radiative loss, as it is typically present in electronic systems. First experiments in this direction include the creation of a heat engine [13] as well as local probes for thermometry in ultracold gases [14-16]. Moreover, the implementation of a heat pump could be also of practical use for reaching lower temperatures.

In this paper, we design, characterize, and propose to implement a quantum heat pump in an optically microstructured quantum gas. The starting point is a setup as it is realized by the Zurich group, where two reservoirs are coupled by

\footnotetext{
*arko.roy@unitn.it

†eckardt@tu-berlin.de
}

Published by the American Physical Society under the terms of the Creative Commons Attribution 4.0 International license. Further distribution of this work must maintain attribution to the author(s) and the published article's title, journal citation, and DOI. Open access publication funded by the Max Planck Society. a structured channel [13,17-19]. The device itself is based on the potential landscape sketched in Fig. 1(a). It consists of two coupled quantum dots, to be labeled $l$ (left) and $r$ (right), each coupled to a larger fermionic system and each hosting two relevant single-particle levels, to be labeled by 1 (lower) and 2 (upper) [Fig. 1(a)]. The upper levels shall correspond to excitations transverse to the sketched potential. This brings various advantages: the energies of the individual levels can easily be tuned by the local transverse potential, the tunnel coupling between the upper levels is comparable to that of the lower ones, and unwanted tunneling between the upper and lower level of different dots is forbidden by opposite transverse parity. Finally, we assume a time-periodic energy modulation $K \cos (\omega t)$ for particles in the right dot with amplitude $K$ and frequency $\omega$. The proposed setup can be realized using available experimental techniques: Two fermionic reservoirs coupled by tailor-made potential landscapes have already been realized by the Zurich group [13,1719]. Furthermore, the combination of digital mirror devices with high-resolution optics has become a standard technique for engineering light-shift potentials with high spatiotemporal resolution [8].

A driven double-quantum-dot structure similar to the one considered here was proposed also for electrons in a driven heterostructure [20]. It was modeled as a one-dimensional system with stepwise constant potentials, which allowed for a treatment using a Floquet transfer-matrix approach [21] and which differs from the system considered here by the fact that it does not involve transverse degrees of freedom. Different from this previous work, our analysis presented below is based on Floquet-Born-Markov theory [22-25]. Another difference consists in the fact that we are assuming reservoirs of finite size, as they are relevant for a quantum-gas system, and study their time evolution. The latter reveals the interplay between cooling via energy pumping on the one hand, which for (suitable parameters) dominates on short times, and heating that we relate to driving induced micromotion on the other, 

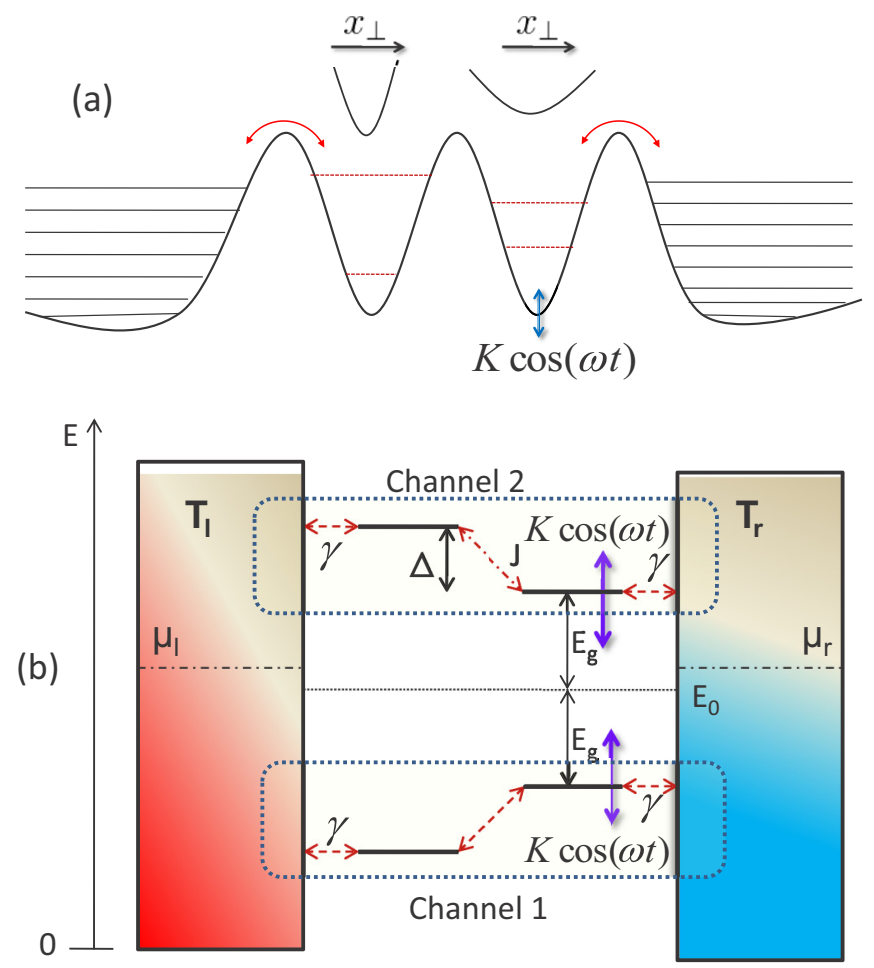

FIG. 1. (a) Sketch of the potential landscape used to build the heat pump. (b) Open-system model used to describe the device.

which eventually will dominate in the long-time limit. The fact that the working mechanism of our system will be based on the transport of particles (fermions) between the dots and the reservoirs, distinguishes it, moreover, from very recently proposed periodically driven heat pumps based on energy exchange $[26,27]$ and from an incoherently driven double-dot device [28].

\section{BASIC IDEA}

The model that we employ to describe the system is sketched in Fig. 1(b). We treat the fermionic systems coupled to the left and right quantum dot as thermal reservoirs characterized by temperatures $T_{l}, T_{r}$ and chemical potentials $\mu_{l}, \mu_{r}$. For simplicity, we assume (i) the reservoirs (which we label by the dot they are coupled to, $d=l, r$ ) to have a density of states $D_{d}(E)=\rho_{d} \theta(E)$ taking a constant value $\rho_{d}$ above their minimum energy $E=0$ ( $\theta$ denotes the step function) and (ii) the particle exchange between reservoirs and dots via tunneling to be captured by an energy independent parameter $\gamma$. However, this choice is not essential.

Within the double-dot structure, for zero driving $(K=0)$ the lower and the upper state shall be arranged symmetrically with respect to the energy $E_{0}$ in both wells and they shall be separated by $2\left(E_{g}+\Delta\right)$ and $2 E_{g}$ in the left and the right well, respectively, with $\Delta, E_{g}>0$. Moreover, particles can tunnel "horizontally" between the lower and the upper pair of levels with matrix element $-J$, whereas "diagonal" tunneling between the lower level of one dot to the upper one of the other dot is suppressed by the opposite parity of the transverse wave function. Thus, the lower and the upper pair of levels form two individual channels, 1 and 2, each connecting both reservoirs. All in all, the double-dot system is described by the Hamiltonian

$$
\begin{aligned}
\hat{H}(t)= & \left(E_{0}+E_{g}+\Delta\right) \hat{n}_{2 l}+\left[E_{0}+E_{g}+K \cos (\omega t)\right] \hat{n}_{2 r} \\
& +\left(E_{0}-E_{g}-\Delta\right) \hat{n}_{1 l}+\left[E_{0}-E_{g}+K \cos (\omega t)\right] \hat{n}_{1 r} \\
& -J\left(\hat{a}_{1 r}^{\dagger} \hat{a}_{1 l}+\hat{a}_{2 r}^{\dagger} \hat{a}_{2 l}+\text { H.c. }\right)
\end{aligned}
$$

where H.c. stands for hermitian conjugate and $\hat{a}_{c d}$ and $\hat{n}_{c d}$, with dot label $d=l, r$ and channel label $c=1,2$, denote the annihilation and number operators for spinless fermions in the four levels.

In the following, we will assume $J \ll \Delta$, so that tunneling between the dots is energetically suppressed in the undriven system. However, we will also assume that the driving frequency is tuned to resonance with this offset, $\Delta=v \hbar \omega$ with integer $v$, so that coherent tunneling can be induced as a "photon"-assisted process. In this way, one can achieve a situation, where fermions of energy $E_{0}+E_{g}$ absorb $v$ energy quanta $\hbar \omega$ from the drive, when passing from the right to the left reservoir via channel 2, whereas fermions at the lower energy $E_{0}-E_{g}$ emit the energy $\nu \hbar \omega$ into the drive, when moving from right to left via channel 1 . Together with the reverse left-to-right processes, one can immediately see that a steady-state situation without net particle and energy flow between both reservoirs can be given by a configuration, where $\mu_{l}=\mu_{r}=E_{0}$ and

$$
\frac{T_{l}}{T_{r}}=\frac{E_{g}+\Delta}{E_{g}} \equiv a>1 .
$$

Namely, in this case the Fermi-Dirac distribution $f_{T, \mu}(E)=$ $\{\exp [(E-\mu) / T]+1\}^{-1}$ of the right reservoir at energies $E_{0} \pm E_{g}$ equals that of the left reservoir at energies $E_{0} \pm\left(E_{g}+\right.$ $\Delta)$, respectively. Therefore, one can expect that the driven double-dot acts as a quantum heat pump, transferring energy from the colder right to the hotter left reservoir, as long as

$$
\frac{T_{l}}{a} \lesssim T_{r}<T_{l}
$$

Note that Eqs. (2) and (3) are simple estimates, only. Deviations from them will arise due to "photon"-assisted tunneling processes between the levels $E_{0} \pm E_{g}$ of the driven right quantum dot and states of the right reservoir at energies $E_{0} \pm$ $E_{g}+m \hbar \omega$, during which integer numbers $m \neq 0$ of energy quanta $\hbar \omega$ are absorbed or emitted by the drive. Furthermore, also the level splitting within each channel, as it is induced by resonant tunneling, alters this simple picture.

\section{OPEN-SYSTEM APPROACH}

Each channel corresponds to a driven two-level system. In the "rotating" reference frame, obtained by integrating out the potential offsets $\hat{V}=\sum_{c} s_{c} \nu \hbar \omega \hat{n}_{c l}+K \cos (\omega t) \hat{n}_{c r}$ between both wells of each channel, with sign $s_{c} \equiv(-1)^{c}$, the tunneling parameter becomes time dependent. Namely, after a gauge transformation $\hat{H}^{\prime}=\hat{U}^{\dagger} \hat{H} \hat{U}-i \hbar \hat{U}^{\dagger} \frac{d}{d t} \hat{U}$ with the time-periodic unitary operator $\hat{U}(t)=\exp \left[-i \int_{0}^{t} d t \hat{V}(t) / \hbar\right]$, 
we arrive at

$$
\begin{aligned}
\hat{H}^{\prime}(t)= & \sum_{c=1,2}\left[\left(E_{0}+s_{c} E_{g}\right) \hat{n}_{c}\right. \\
& \left.-J\left(e^{i\left(\alpha \sin (\omega t)-s_{c} \nu \hbar \omega t\right)} \hat{a}_{c r}^{\dagger} \hat{a}_{c l}+\text { H.c. }\right)\right],
\end{aligned}
$$

where we have introduced the total channel occupations $\hat{n}_{c}=$ $\hat{n}_{c l}+\hat{n}_{c r}$ as well as the dimensionless driving amplitude $\alpha \equiv$ $K /(\hbar \omega)$. In the high-frequency limit $J \ll \hbar \omega$, we can average this rapidly varying phase factor over one driving period to obtain the effective time-independent Hamiltonian in rotatingwave approximation,

$$
\hat{H}_{\mathrm{eff}}=\sum_{c=1,2}\left[\left(E_{0}+s_{c} E_{g}\right) \hat{n}_{c}-J_{c}^{\mathrm{eff}}\left(\hat{a}_{c r}^{\dagger} \hat{a}_{c l}+\text { H.c. }\right)\right] .
$$

Here, $J_{c}^{\text {eff }}=J \mathcal{J}_{s_{c} v}(\alpha)$ denotes the effective tunneling matrix element, where $\mathcal{J}_{k}(x)$ denotes the $k$ th-order Bessel function of the first kind (see, e.g., Ref. [29] for details). Diagonalizing $\hat{H}_{\text {eff }}$ Hamiltonian and transforming the eigenstates back to the original frame of reference, one obtains the time-periodic single-particle Floquet modes $\left|u_{c \pm}(t)\right\rangle=$ $\left(e^{-i s_{c} \nu \omega t}|c l\rangle \pm e^{-i \alpha \sin (\omega t)}|c r\rangle\right) / \sqrt{2}$, with quasienergies $\varepsilon_{c \pm}=$ $E_{0}+s_{c} E_{g} \pm J_{c}^{\text {eff }}$, where $|c d\rangle=\hat{a}_{c d}^{\dagger} \mid$ vac $\rangle$ with vacuum $|\mathrm{vac}\rangle$. For noninteracting fermions, we also define time-periodic Floquet-Fock states $\left|\left\{n_{c \pm}\right\}(t)\right\rangle$ characterized by sharp occupation numbers $n_{c \pm}$ of the single-particle Floquet modes.

Let us treat the double-quantum dot as an open system coupled to the left and the right reservoir [Fig. 1(b)]. In the limit where the coupling to the reservoirs, $\gamma$, becomes small compared to the quasienergy level splitting $\sim\left|J_{c}^{\text {eff }}\right|$ ), the system approaches a quasisteady state described by a time-periodic density matrix $\hat{\rho}(t)=\sum_{\left\{n_{c \pm}\right\}} p_{\left\{n_{c \pm}\right\}}\left|\left\{n_{c \pm}\right\}(t)\right\rangle\left\langle\left\{n_{c \pm}\right\}(t)\right|$, which is diagonal with respect to the time-periodic Floquet-Fock states [22-25]. The diagonal elements are given by timeindependent probabilities $p_{\left\{n_{c \pm}\right\}}$, which are determined by the rates $\left(1-n_{c \pm}\right) R_{c \pm}^{*}$ and $n_{c \pm} R_{c \pm}^{\dagger}$ for the gain ("birth" $*$ ) and the loss ("death" $\dagger$ ) of a fermion in state $c \pm$, respectively. The rates have contributions from both reservoirs $(d=l, r)$, $R_{c \pm}^{\eta}=R_{c \pm}^{\eta l}+R_{c \pm}^{\eta r}$ with $\eta=*, \dagger$, and can be obtained using Floquet-Born-Markov theory in combination with the secular approximation [22-25]. They are given by a sum of goldenrule type terms describing processes where the system exchanges $m$ energy quanta $\hbar \omega$ with the drive, $R_{c \pm}^{\eta d}=\sum_{m} R_{c \pm}^{\eta d(m)}$ with

$$
R_{c \pm}^{\eta d(m)}=\frac{2 \pi}{\hbar}\left|\gamma_{c d, \pm}^{(m)}\right|^{2} D_{d}\left(\varepsilon_{c \pm}+m \hbar \omega\right) f_{T_{d}, \mu_{d}}^{\eta}\left(\varepsilon_{c \pm}+m \hbar \omega\right),
$$

where $f_{T, \mu}^{*}(E)=f_{T, \mu}(E), f_{T, \mu}^{\dagger}(E)=1-f_{T, \mu}(E)$ as well as $\gamma_{c d, \pm}^{(m)}=\frac{1}{T} \int_{0}^{T} \mathrm{~d} t e^{i m \omega t}\left\langle\operatorname{vac}\left|\gamma \hat{a}_{c d}\right| u_{c \pm}(t)\right\rangle$. For the undriven left dot only one term of the sum contributes, $\gamma_{c l, \pm}^{(m)}=$ $\gamma \delta_{m+s_{c} \nu} / \sqrt{2}$, describing the coupling to reservoir states at energies $E_{0}+s_{c}\left(E_{g}+\Delta\right) \pm J_{c}^{\text {eff }}$. In contrast for the driven right dot, we find coupling matrix elements $\gamma_{c r, \pm}^{(m)}=\gamma \mathcal{J}_{m}(\alpha) / \sqrt{2}$ for particle exchange with reservoir states at all energies $E_{0}+s_{c} E_{g} \pm J_{c}^{\text {eff }}+m \hbar \omega$. The "satellite" coupling terms with nonzero $m$ are a direct consequence of the periodic time-dependence of the Floquet states $\left|u_{c \pm}(t)\right\rangle$ known as micromotion.

For small driving amplitudes one has $\mathcal{J}_{m}(\alpha) \simeq(\alpha / 2)^{m} / m$ !. Thus, the ideal situation captured by Eqs. (2) and (3), where the right dot is just coupled to reservoir states of energy $E_{0}+s_{c} E_{g}$, is given in the limit $\alpha \rightarrow 0$, only. However, in this limit also the effective tunneling matrix elements vanish, $J_{c}^{\text {eff }} \rightarrow 0$, suppressing transport between both reservoirs. As a result, when choosing $\alpha$ there will be a trade-off between enhancing $J_{c}^{\text {eff }}$ on the one hand and avoiding detrimental processes associated with photon-assisted $(m \neq 0)$ tunneling between the system and the right reservoir on the other hand. Since our theory is limited to the regime $\gamma \ll\left|J_{c}^{\text {eff }}\right|$, where the bottleneck of transport from one reservoir to the other through the double dot is $\gamma$ rather than $J_{c}^{\text {eff }}$, it does not directly describe the suppression of transport for $\alpha \rightarrow 0$. However, it is still taken into account indirectly by the fact that transport is limited by a value of $\gamma$, which is assumed to be smaller than $\left|J_{c}^{\text {eff }}\right|$.

In the steady state the mean-occupations of the Floquet modes obey $\frac{\mathrm{d}}{\mathrm{d} t}\left\langle\hat{n}_{c \pm}\right\rangle=R_{c \pm}^{*}\left(1-\left\langle\hat{n}_{c \pm}\right\rangle\right)-R_{c \pm}^{\dagger}\left\langle\hat{n}_{c \pm}\right\rangle=0$, so that $\left\langle\hat{n}_{c \pm}\right\rangle=\left(1+R_{c \pm}^{\dagger} / R_{c \pm}^{*}\right)^{-1}$. From this solution, we obtain the steady-state rates for the change of particle number, $\dot{N}_{d}$, and energy, $\dot{E}_{d}$, in both reservoirs:

$$
\begin{aligned}
& \dot{N}_{d}=\sum_{c \pm}\left[R_{c \pm}^{\dagger d}\left\langle\hat{n}_{c \pm}\right\rangle-R_{c \pm}^{* d}\left(1-\left\langle\hat{n}_{c \pm}\right\rangle\right)\right], \\
& \dot{E}_{d}=\sum_{\substack{c \pm \\
m}}\left[R_{c \pm}^{\dagger d(m)}\left\langle\hat{n}_{c \pm}\right\rangle-R_{c \pm}^{* d(m)}\left(1-\left\langle\hat{n}_{c \pm}\right\rangle\right)\right]\left(\varepsilon_{c \pm}+m \hbar \omega\right) .
\end{aligned}
$$

While the total particle number is conserved, so that $\dot{N}_{r}+$ $\dot{N}_{l}=0$, energy is not conserved for nonzero driving, so that $\dot{E}_{r}+\dot{E}_{l} \neq 0$ (we always find $\dot{E}_{r}+\dot{E}_{l}>0$, consistent with the second law of thermodynamics). In the quantum-heat-pump regime, $\dot{E}_{r}<0$ for $T_{r}<T_{l}$, where the system extracts energy from the colder right reservoir, we can define the coefficient of performance COP $=-\dot{E}_{r} /\left(\dot{E}_{r}+\dot{E}_{l}\right)$.

\section{RESERVOIR DYNAMICS}

Assuming the reservoirs to thermalize sufficiently fast to individually remain in equilibrium while exchanging energy and particles with the double dot, we compute the time derivatives of $\mu_{d}$ and $T_{d}$ from Eqs. (7). For this purpose, we invert

$$
\left(\begin{array}{c}
\dot{N}_{d} \\
\dot{E}_{d}
\end{array}\right)=\left(\begin{array}{cc}
\partial_{\mu_{d}} N_{d} & \partial_{T_{d}} N_{d} \\
\partial_{\mu_{d}} E_{d} & \partial_{T_{d}} E_{d}
\end{array}\right)\left(\begin{array}{c}
\dot{\mu}_{d} \\
\dot{T}_{d}
\end{array}\right)
$$

where $N_{d}=N_{d}\left(T_{d}, \mu_{d}\right)=\int_{0}^{\infty} \mathrm{d} \epsilon \rho_{d} f_{T_{d}, \mu_{d}}(\epsilon)$ and $E_{d}=$ $E_{d}\left(T_{d}, \mu_{d}\right)=\int_{0}^{\infty} \mathrm{d} \epsilon \rho_{d} \epsilon f_{T_{d}, \mu_{d}}(\epsilon)$. In the following, we will use the tunneling parameter $J$ as the unit of energy and measure times in units of $\tau=\hbar /\left(2 \pi \gamma^{2} \rho_{r}\right)$, so that $\gamma$ drops out and the dynamics depends on the ratio $\lambda \equiv \rho_{l} / \rho_{r}$ of reservoir "sizes" rather than on the absolute values $\rho_{r}$ and $\rho_{l}$.

\section{RESULTS}

In order to test, whether the driven double dot can operate as a heat pump, in Fig. 2(a), we examine the rate $\dot{T}_{r}$ at which 

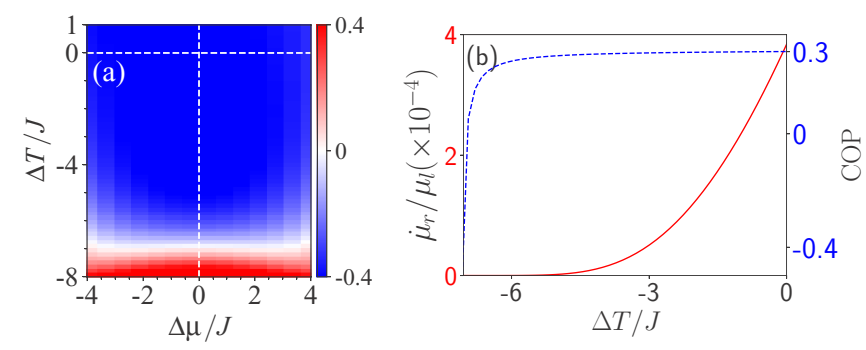

FIG. 2. (a) Change rate of the right temperature, $\dot{T}_{r}$, vs. $\Delta T=$ $T_{r}-T_{l}$ and $\Delta \mu=\mu_{r}-\mu_{l}$, for fixed $\mu_{l}=E_{0}=46$ and $T_{l}=0.2 \mu_{l}$ and $\alpha=0.1, \Delta=\hbar \omega=20, E_{g}=6, \lambda=10$. (b) Relative change rate of right chemical potential $\dot{\mu}_{r} / \mu_{r}$ (red solid line, left axis) and coefficient of performance COP vs. $\Delta T$ (blue dotted line, right axis) for $\Delta \mu=0$ for parameters of (a).

the right temperature changes in response to finite differences in temperature and chemical potential, $\Delta T=T_{r}-T_{l}$ and $\Delta \mu=\mu_{r}-\mu_{l}$, keeping $T_{l}$ and $\mu_{l}$ fixed (the parameters are given in the caption). Since we are considering a small driving amplitude $\alpha=0.1$, for $\Delta \mu=0$ we expect the system to operate as a heat pump roughly in the regime (3), i.e., for $0>\Delta T>-(1-1 / a) T_{l} \approx-7.1$. And, indeed, we find $\dot{T}_{r}<0$ for $\Delta T \gtrsim-7$, in excellent agreement with this prediction. Moreover, the relative rate at which the right chemical potential changes [Fig. 2(b), red line] is negligibly small, as desired for the operation of the device as a heat pump. The blue line in Fig. 2(b) shows the coefficient of performance, reaching its maximum of about 0.3 at $\Delta T=0$.

In Fig. 3(a), we plot the time evolution of $T_{r}$ (solid lines) and $T_{l}$ (long-dashed lines) starting from the same initial temperature $T_{0}$ and chemical potential $\mu_{0}=E_{0}$ (the parameters are given in the caption). The thin green, intermediate blue, and thick red lines correspond to increasing driving strengths $\alpha=0.1,0.25$, and 0.5 , respectively. The best performance can be observed for the weakest driving strength. Here the temperature of the left reservoir first drops to $0.6 T_{0}$ due to heat transfer to the left reservoir, before it increases again very slowly as a result of driving induced heating. Increasing $\alpha$ and with that also $\left|J_{c}^{\text {eff }}\right|$ the dynamics can be made faster by increasing $\gamma$ so that $\tau$ becomes smaller. However, at the same time also driving-induced heating increases with $\alpha$ and causes a smaller temperature reduction to $0.8 T_{0}$ for $\alpha=0.25$ and even a temperature increase of both reservoirs for $\alpha=0.5$. The inset shows the time evolution of the chemical potential $\mu_{r}$ of the right reservoir for $\alpha=0.25$. We can see that it changes by about one percent only on the time scale needed to reach the minimum temperature, so that the driven double dot predominantly cools the right reservoir.

The driving-induced heating is directly associated with the micromotion and the resulting rates $R_{c \pm}^{\eta d(m)}$ with $m \neq 0$ [Eq. (6)]. When we set the $m \neq 0$ rates to zero artificially by hand, no driving-induced heating occurs and a steady state with $T_{r} \approx 0.54 T_{0}$ is reached, as can be seen from the thin short-dashed red line showing $T_{r}$ for $\alpha=0.5$ (where before no cooling was observed at all). For this artificial steady state, we expect $T_{l} / T_{r} \approx 1+\Delta / E_{g}=a$, according to Eq. (2). Including also $m \neq 0$, this estimate sets also an upper limit for $T_{l} / T_{r}$ at the time where $T_{r}$ becomes minimal. This is confirmed in
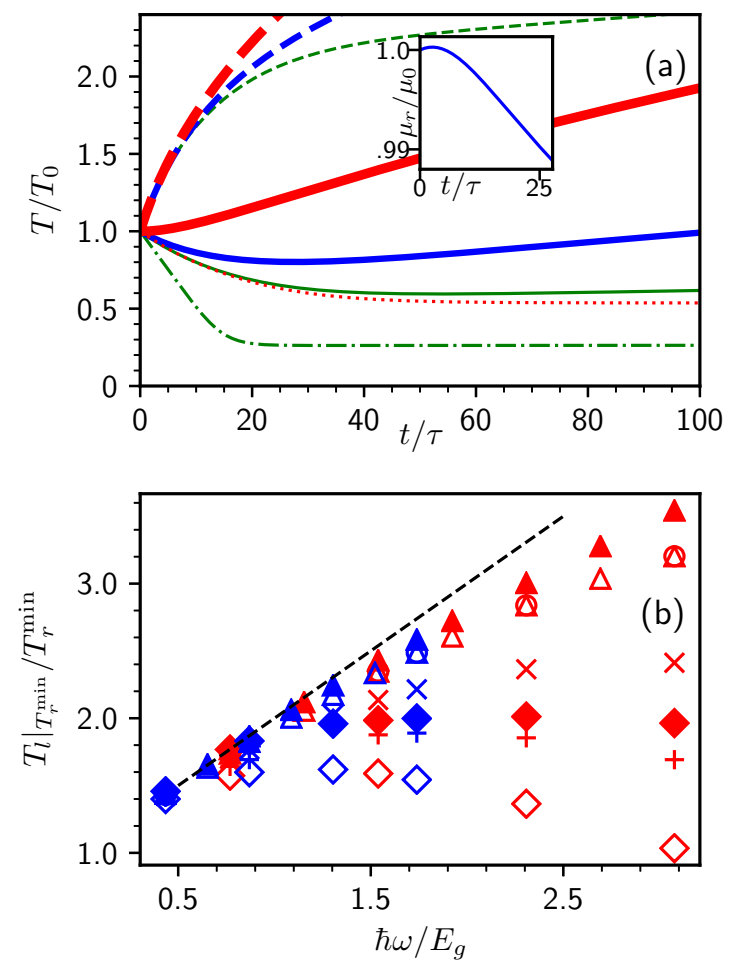

FIG. 3. (a) Time evolution of $T_{r}$ (solid lines) and $T_{l}$ (dashed lines) for initial conditions $T_{r}=T_{l}=T_{0}=0.2 \mu_{l}$ and $\mu_{l}=\mu_{r}=E_{0}=46$; driving amplitudes $\alpha=0.1$ (thin green), 0.25 (intermediate blue), 0.5 (thick red); as well as for $E_{g}=6, \Delta=\hbar \omega=20$ (giving $a=13 / 3$ ), and $\lambda=1$. Dotted red line shows $T_{r}$ for $\alpha=0.5$ neglecting rates $R_{c \pm}^{\eta d(m)}$ with $m \neq 0$. Dot-dashed green line shows $T_{r}$ for $\alpha=0.1$ and $\lambda=10$. Inset: Evolution of right chemical potential for $\alpha=0.25$. (b) $T_{l} / T_{r}$ when $T_{l}$ reaches its minimum vs. $\hbar \omega / E_{g}$; for $E_{g}=6$ (red symbols), $=12$ (blue symbols); different $\hbar \omega=\Delta ; \alpha=0.1$ (triangles), 0.2 (circles), 0.3 (diagonal crosses), 0.4 (pluses), 0.5 (diamonds); $\lambda=10$ (filled symbols), 1 (open and other symbols). The dashed line shows $a$.

Fig. 3(b), where we plot this ratio versus $\Delta / E_{g}=\hbar \omega / E_{g}$ for various different parameters (different symbols, see caption) together with $a$ (dashed line). From this figure, we can see

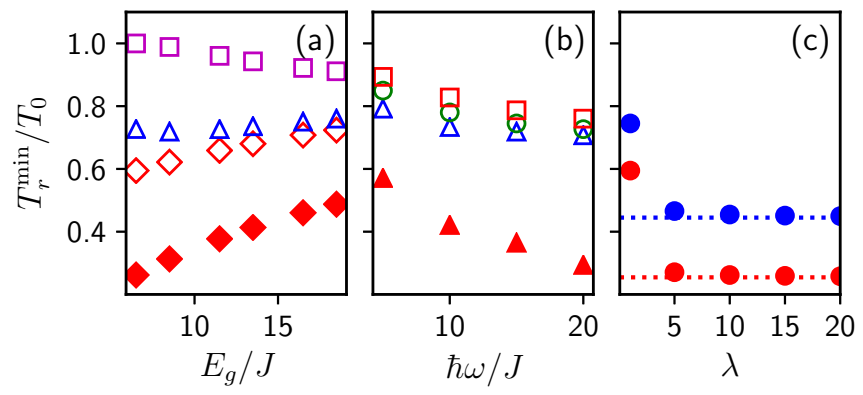

FIG. 4. $T_{r}^{\min } / T_{0}$ for initial conditions $T_{r}=T_{l}=T_{0}=0.2 \mu_{l}$ and $\mu_{l}=\mu_{r}=E_{0}=46$ (a) vs. $E_{g}$ for $\hbar \omega / J=20 ; \alpha=0.1$ (diamonds), 0.2 (triangles), 0.5 (squares); $\lambda=1$ (open symbols), 10 (filled symbols); (b) vs. $\hbar \omega=\Delta$ for $\alpha=0.2 ; E_{g}=6$ (triangles), 12 (circles), 18 (squares); $\lambda=1$ (open symbols), 10 (filled symbols); (c) vs. $\lambda$ for $E_{g}=6$ for $\omega=\Delta=20, \alpha=0.1$ (lower red circles) and $E_{g}=12$, $\omega=\Delta=15, \alpha=0.2$ (upper blue circles); the dashed lines show the corresponding results for $\lambda \rightarrow \infty$. 


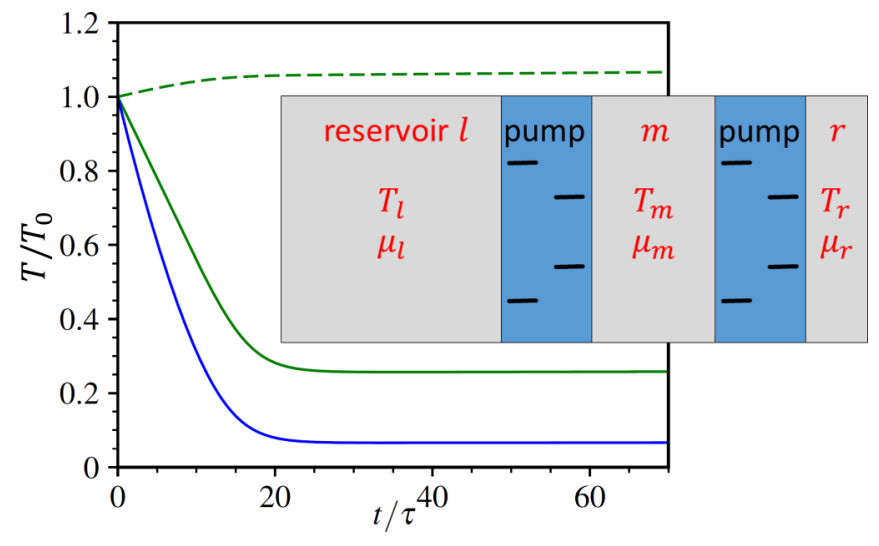

FIG. 5. Temperature evolution of three reservoirs $l, m, r$ (upper, middle, lower lines) coupled by identical heat pumps; for initial conditions $T_{l}=T_{m}=T_{r}=T_{0}=0.2 \mu_{r}, \mu_{l}=\mu_{m}=\mu_{r}=E_{0}=46$ and $\hbar \omega=\Delta=20, E_{g}=6, \alpha=0.1, \rho_{l} / \rho_{m}=\rho_{m} / \rho_{r}=5$.

that, as expected, the data come closer to the optimal limit $a$ (dashed line) when $\alpha$ is lowered. While also lowering $\hbar \omega / E_{g}$ helps to approach the bound $a$, the bound itself becomes more favorable for larger $\hbar \omega / E_{g}$, so that (at least for sufficiently small $\alpha$ ) we still find better (larger) temperature ratios for larger $\hbar \omega / E_{g}$.

Finally, we observe larger temperature ratios when increasing the relative size $\lambda$ of the energy absorbing left bath. This is a consequence of the fact that the left bath can absorb more of the heating-induced energy during the evolution, since its temperature remains lower. An additional major benefit of raising $\lambda$ is, furthermore, that not only $T_{r} / T_{l}$ is lowered, but at the same time also the absolute values of $T_{l}$ and with this also $T_{r}$. The advantage of an increased left reservoir is confirmed by the dash-dotted green line in Fig. 3(a) showing the evolution of $T_{r}$ for the same parameters like the solid green curve, except that now $\lambda=10$ is chosen rather than the value $\lambda=1$ used for all other curves. As a result, $T_{r}$ is reduced to $0.26 T_{0}$, which is almost the optimal value $T_{0} / a \approx 0.23 T_{0}$ of the bound

$$
T_{r} \gtrsim T_{0} / a
$$

obtained from Eq. (3) for $\lambda \rightarrow \infty$ so that $T_{l}=T_{0}$ at all times.

In Fig. 4 we investigate how the minimal right temperature assumed during the evolution depends on the system parameters. As expected from Eq. (2), we find that $T_{r}^{\min } / T_{0}$ decreases both when lowering $E_{g}$ [at least for sufficiently weak driving strength $\alpha$, Fig. 4(a)] and when increasing $\hbar \omega=\Delta$ [Fig. 4(b)]. Moreover, we find that the enhancement of cooling by increasing the relative size $\lambda$ of the right reservoir saturates at values of about $\lambda=5$ [Fig. 4(c)]. This is a very promising result for the implementing such a heat pumps with ultracold atoms, since it implies that it is sufficient to engineer reservoirs of rather moderate size compared to the subsystem to be cooled down.

Besides the optimization of parameters with the aims of both saturating and lowering the bound (9), an alternative strategy for reaching lower temperatures is to put two (or more) heat pumps in series. This scenario is investigated in Fig. 5, where we plot the evolution of the temperatures of three reservoirs $(l, m, r)$ that are coupled by two identical heat pumps (see sketch). Motivated by the results of Fig. 4(c), we have chosen a hierarchy of reservoir sizes according to $\rho_{l} / \rho_{m}=\rho_{m} / \rho_{r}=5$. Note that both left and middle reservoir together are still only 30 times larger than the reservoir that we wish to cool. The lower limit for the temperature of the right reservoir is now given by $T_{r} \gtrsim T_{0} / a^{2} \approx 0.053 T_{0}$ and, indeed, we can see that during the evolution $T_{r} / T_{0}$ is reduced to the temperature $0.07 T_{0}$, which is only slightly larger.

\section{CONCLUSION}

We have described a simple design for a quantum heat pump. It is based on two coherently coupled, periodically driven quantum dots, each possessing two relevant quantum states and being tunnel-coupled to a reservoir. The working principle is based on energy-selective "photon"-assisted tunneling processes, where a particle that tunnels from one dot to the other either absorbs or emits the energy quantum $\hbar \omega$ associated with the driving frequency, depending on its energy. We simulate the device using an open-system approach based on Floquet-Born-Markov theory and show that it indeed works as a heat pump and that unavoidable fundamental driving-induced heating is directly linked to the micromotion of the system's Floquet states. It is a promising perspective to implement such a device with quantum-gases using recently established experimental techniques for microstructuring light-shift potentials. Namely, quantum gases provide extremely clean conditions, since they do not suffer from detrimental dissipation via radiative losses or the coupling to a phonon-bath, as it is present in electronic systems. Moreover, the proposed device might also be of practical use for reaching lower temperatures.

\section{ACKNOWLEDGMENTS}

We acknowledge insightful discussions with M. Alamo, M. Lebrat, L. Corman, and D. Vorberg. This research was funded by the Deutsche Forschungsgemeinschaft (DFG) via the Research Unit FOR 2414 under Project No. 277974659.
[1] R. Kosloff and A. Levy, Annual Review of Physical Chemistry 65, 365 (2014).

[2] G. Benenti, G. Casati, K. Saito, and R. Whitney, Phys. Rep. 694, 1 (2017).

[3] M. Campisi, P. Hänggi, and P. Talkner, Rev. Mod. Phys. 83, 771 (2011).
[4] S. Vinjanampathy and J. Anders, Contemp. Phys. 57, 545 (2016).

[5] F. Brandão, M. Horodecki, N. Ng, J. Oppenheim, and S. Wehner, Proc. Natl. Acad. Sci. USA 112, 3275 (2015).

[6] J. Gemmer, M. Michel, and G. Mahler, Quantum Thermodynamics: Emergence of Thermodynamic Behavior Within 
Composite Quantum Systems, Lecture Notes in Physics (Springer, Berlin/Heidelberg, 2004).

[7] H. Ott, Rep. Prog. Phys. 79, 054401 (2016).

[8] S. Kuhr, Nat. Sci. Rev. 3, 170 (2016).

[9] L. W. Cheuk, M. A. Nichols, M. Okan, T. Gersdorf, V. V. Ramasesh, W. S. Bakr, T. Lompe, and M. W. Zwierlein, Phys. Rev. Lett. 114, 193001 (2015).

[10] A. Alberti, C. Robens, W. Alt, S. Brakhane, M. Karski, R. Reimann, A. Widera, and D. Meschede, New J. Phys. 18, 053010 (2016).

[11] M. McDonald, J. Trisnadi, K.-X. Yao, and C. Chin, Phys. Rev. X 9, 021001 (2019).

[12] A. Mazurenko, S. Blatt, F. Huber, M. F. Parsons, C. S. Chiu, G. Ji, D. Greif, and M. Greiner, Rev. Sci. Instrum. 90, 033101 (2019).

[13] J.-P. Brantut, C. Grenier, J. Meineke, D. Stadler, S. Krinner, C. Kollath, T. Esslinger, and A. Georges, Science 342, 713 (2013).

[14] D. Mayer, F. Schmidt, S. Haupt, Q. Bouton, D. Adam, T. Lausch, E. Lutz, and A. Widera, arXiv:1901.06188.

[15] Q. Bouton, J. Nettersheim, D. Adam, F. Schmidt, D. Mayer, T. Lausch, E. Tiemann, and A. Widera, Phys. Rev. X 10, 011018 (2020).

[16] M. Hohmann, F. Kindermann, T. Lausch, D. Mayer, F. Schmidt, and A. Widera, Phys. Rev. A 93, 043607 (2016).
[17] S. Krinner, D. Stadler, D. Husmann, J.-P. Brantut, and T. Esslinger, Nature 517, 64 (2014).

[18] S. Krinner, T. Esslinger, and J.-P. Brantut, J. Phys.: Cond. Matt. 29, 343003 (2017).

[19] S. Häusler, S. Nakajima, M. Lebrat, D. Husmann, S. Krinner, T. Esslinger, and J.-P. Brantut, Phys. Rev. Lett. 119, 030403 (2017).

[20] M. Rey, M. Strass, S. Kohler, P. Hänggi, and F. Sols, Phys. Rev. B 76, 085337 (2007).

[21] M. Wagner, Phys. Rev. A 51, 798 (1995).

[22] R. Blümel, A. Buchleitner, R. Graham, L. Sirko, U. Smilansky, and H. Walther, Phys. Rev. A 44, 4521 (1991).

[23] S. Kohler, T. Dittrich, and P. Hänggi, Phys. Rev. E 55, 300 (1997).

[24] D. W. Hone, R. Ketzmerick, and W. Kohn, Phys. Rev. E 79, 051129 (2009).

[25] D. Vorberg, W. Wustmann, H. Schomerus, R. Ketzmerick, and A. Eckardt, Phys. Rev. E 92, 062119 (2015).

[26] C. Charalambous, M. A. Garcia-March, M. Mehboudi, and M. Lewenstein, New J. Phys. 21, 083037 (2019).

[27] A. Riera-Campeny, M. Mehboudi, M. Pons, and A. Sanpera, Phys. Rev. E 99, 032126 (2019).

[28] B. Cleuren, B. Rutten, and C. Van den Broeck, Phys. Rev. Lett. 108, 120603 (2012).

[29] A. Eckardt, Rev. Mod. Phys. 89, 011004 (2017). 